AGATA RĘBKOWSKA

ORCID: 0000-0001-6697-2361

Université de Wrocław

agata.rebkowska@uwr.edu.pl

\title{
ENTRE LE SACRÉ ET LE PROFANE. LE DISCOURS JOURNALISTIQUE FRANÇAIS SUR L'AFFAIRE PUSSY RIOT
}

Le 21 février 2012, trois femmes du groupe punk rock Pussy Riot ont organisé dans la cathédrale du Christ-Sauveur à Moscou une performance intitulée "Vierge Marie, chasse Poutine ». À la suite de cette exhibition dans un lieu de culte orthodoxe, les trois femmes sont condamnées à deux ans de colonie pénitentiaire à régime ordinaire.

Le verdict du tribunal de Moscou, qualifiant cet acte de « vandalisme » et « incitation à la haine religieuse » a encouragé l'opinion publique à se pencher sur le rôle du religieux dans l'État laïque qu'est la Russie.

La presse écrite a cherché à expliquer cet événement à ses lecteurs et à lui donner du sens. Sans compter les genres éditoriaux ou d'innombrables interviews d'experts qui affichaient ouvertement leur prise de position, la presse générale d'information a aussi reflété, par le simple fait de nommer l'événement, un certain point de vue anthropologique sur celui-ci ${ }^{1}$

En prenant en compte les divergences de sens au sein de différentes communautés discursives, nous nous proposons d'examiner le sens social de cet événement construit et transmis dans le discours journalistique français. L'aspect qui nous préoccupe davantage est lié à la place de la religion dans le débat public : le discours journalistique occidental reflète-t-il les tendances désécularisantes de la

${ }^{1}$ Cf. M. Veniard, La nomination des événements dans la presse. Essai de sémantique discursive, Presses universitaires de Franche-Comté, Besançon 2013, p. 7. 
Russie post-soviétique $?^{2}$ Et, le cas échéant, quelles formes prennent-elles dans ce pays, mosaïque d'ethnies et de religions?

Pour examiner le rôle du religieux dans la construction du sens social de la performance du groupe Pussy Riot, nous allons nous servir du concept de paradigme désignationnel qui désigne les « listes de syntagmes (en général nominaux, parfois verbaux) fonctionnant en coréférence avec un vocable initial dans un discours donné $»^{3}$. Ces reformulants, relevant très souvent de la désignation plutôt que de la dénomination, et donc d'un « mode de référenciation alternatif, occasionnel » ${ }^{4}$, ont un impact non négligeable sur les propriétés désignationnelles du vocable initial et contribuent de manière signifiante au processus de construction du sens social du référent ${ }^{5}$.

Dans un premier temps, nous allons donc établir le paradigme désignationnel ${ }^{6}$ constitué de syntagmes renvoyant à un événement réel, à savoir la performance des Pussy Riot dans la cathédrale de Moscou en février 2012. Étant donné le caractère dynamique de la nomination et l'émergence, au fil du temps, de nouvelles étiquettes mettant en lumière des aspects divers, voire contradictoires de l'évènement, l'établissement du paradigme autour du syntagme le plus fréquent et de ses reformulants textuels ne refléterait pas la complexité du phénomène. C'est pourquoi nous adopterons ici une approche onomasiologique en considérant comme constituants du paradigme tous les syntagmes renvoyant à l'événement en question. Nous admettons en même temps l'existence du syntagme de référence - performance — qui, en tant que mot relativement neutre, fréquent et paru dans la presse française le tout premier jour du moment discursif ${ }^{7}$, semble jouer le rôle d'un « hyper-signifiant» structurant les constituants du paradigme. Dans un deuxième temps, nous allons examiner le

${ }^{2} C f$. K.J. Rousselet, « Sécularisation et orthodoxie dans la Russie contemporaine : pour une hypothèse continuiste ? », Questions de Recherche 42, 2013, <https://hal-sciencespo.archives-ouvertes.fr/hal-01070446> [consulté le 10.09.2018].

${ }^{3}$ M.-F. Mortureux, « Paradigmes désignationnels », Semen 8, 1993, <http://journals.openedition.org/semen/4132> [consulté le 10.09.2018].

${ }^{4}$ G. Petit, «La désignation de "timbre-poste" ", Les Carnets du Cediscor 3,1995, <http:// journals.openedition.org/cediscor/475> [consulté le 10.09.2018].

${ }^{5}$ La présente contribution s'inscrit dans l'approche anthropologique de l'acte de nommer. Nous laissons donc de côté la problématique du lien dénominatif (F. Neveu, Dictionnaire des sciences du langage, Armand Colin, Paris 2004, p. 92), c'est-à-dire les désignations ou les dénominations vues comme une relation entre le signe et la réalité. En nous inscrivant dans le versant sémantico-énonciatif lié à la description du langage en usage (M. Veniard, La nomination des événements dans la presse, Presses Universitaires de Franche-Comté, Besançon 2013, p. 15), nous nous intéresserons au résultat de l'acte de nomination.

${ }^{6}$ La présence des reformulants est signalée en discours par le biais du métalangage (par exemple verbes et noms signifier, désigner, nommer, s'appeler, mot, terme, nom), les procédés typographiques (guillemets, parenthèses), le réseau diaphorique ou enfin par équivalence distributionnelle entre les éléments ( $c f$. M.-F. Mortureux, op. cit.).

${ }^{7}$ Que nous comprenons à l'instar de Sophie Moirand comme la diffusion soudaine d'une intense production discursive dans le domaine médiatique, à propos d'un fait ou d'un évènement, laissant par la suite des traces dans des discours relatifs à d'autres faits ou évènements (S. Moirand, Les discours de la presse quotidienne, Presses Universitaires de France, Paris 2007, p. 4). 
fonctionnement préférentiel des constituants du paradigme, pour passer ensuite à l'observation des relations qu'ils entretiennent entre eux. Le corpus comprend des articles parus dans trois journaux français « de référence » : Le Monde, Le Figaro et Libération. Nous nous sommes concentrés sur la presse écrite en raison du rôle incontestable de cette dernière. Même à l'ère numérique, les journaux nationaux retenus continuent de constituer un point de référence en ce qui concerne le discours médiatique français : non seulement ils sont « un lieu de rencontre » des différents discours, mais les sens qui y sont construits sont aussi véhiculés et reformulés ensuite dans d'autres médias. En outre, le choix de journaux de tendances politiques et culturelles différentes permet de rendre compte des préférences linguistiques représentatives pour l'aire géographique et culturelle qu'est la France ${ }^{8}$.

Les articles, centrés autour de l'événement réel, ont été publiés au moment de la diffusion d'une abondante production médiatique sur ce sujet (mars-août 2012) et traitent de deux étapes de l'événement : la performance et le procès du groupe. Le corpus compte au total 19422 mots 9 .

\section{LA RUSSIE DÉSÉCULARISÉE}

Une réflexion sur la place de la religion dans l'espace public russe d'aujourd'hui est d'autant plus justifiée si l'on prend en compte la dynamique des changements dans le domaine religieux en Russie. Avec le déclin de l'athéisme à la fin de l'époque communiste, on observe un nouvel essor du religieux dans la sphère privée comme dans la sphère publique. De nombreux chercheurs y voient une tendance désécularisante qui serait une réaction à l'athéisation forcée de la société soviétique. D'autres, comme Kathy Rousselet, considèrent ce renouveau religieux non pas comme une rupture, mais, paradoxalement, comme un héritage de l'ancien régime : la forte présence de la religion dans la sphère publique serait simplement signe du remplacement d'un système officiel — ici le soviétisme — par un autre : le christianisme orthodox $\mathrm{e}^{10}$. Quelle que soit la raison de ce phénomène, on ne peut pas nier le resserrement récent des liens entre le séculier et le sacré en Russie. La canonisation du tsar Nicolas II et de sa famille en 2000, la loi de 2010 sur le transfert de plus de 6000 sites religieux à l'Église orthodoxe, la loi de 2012 sur l'enseignement obligatoire du religieux à l'école ${ }^{11}$, la présence du patriarche orthodoxe Alexis II aux

${ }^{8}$ Cf. G. Cislaru, Étude sémantique et discursive du nom de pays dans la presse française avec référence à l'anglais, au roumain et au russe, [thèse de doctorat, non publiée], Paris 2005.

${ }^{9}$ La présente contribution s'inscrit dans un projet plus large consacré à la construction du sens social de la Russie dans le discours journalistique français et polonais. Le présent projet, centré sur le discours français, a été réalisé grâce au soutien du Gouvernement Français et de l'Ambassade de France en Pologne, dans le cadre de la Bourse BGF.

${ }^{10}$ K.J. Rousselet, op. cit.

${ }^{11}$ Décrit comme enseignement « des fondements des cultures religieuses et de l'éthique laïque » (voir <http://www.eurel.info/spip.php?article2402> [consulté le 9.09.2018]). 
cérémonies d'investiture à la présidence de Boris Eltsine ou de Dmitri Medvedev et du patriarche Cyrille à celle de Vladimir Poutine, la loi fédérale de 1997 qui reconnaît le rôle de la religion orthodoxe dans l'histoire du pays, un nombre considérable d'accords entre les institutions ecclésiastiques et étatiques, comme l'armée ${ }^{12}$, seraient la preuve des liens étroits entre le religieux et le politique.

\section{L'ÉVÉNEMENT RÉEL}

C'est le désaccord face à cette connivence supposée de l'Église et du pouvoir et à la politique de Vladimir Poutine, alors en campagne présidentielle, qui ont incité les membres du collectif Pussy Riot à passer à l'action. En décembre 2011, le groupe organise déjà des performances artistiques dans les rues de Moscou. Le discours du patriarche Kirill du 8 février 2012, dans lequel les deux premiers mandats de Poutine sont qualifiés de «miracle de Dieu », est à l'origine directe de la célèbre performance à la cathédrale orthodoxe du Christ-Sauveur. Avec des paroles invitant la Vierge Marie à " chasser Poutine », le collectif exprime son désaccord avec la politique de l'État ${ }^{13}$.

Le 3 mars 2012, les trois activistes du groupe Ekaterina Samoutsevitch, Nadejda Tolokonnikova et Maria Alekhina sont arrêtées par la police. Placées en détention provisoire jusqu'au mois d'août, elles sont ensuite condamnées par un tribunal de Moscou à deux ans de camp. Plusieurs intellectuels et célébrités internationales ont alors exprimé leur soutien aux membres du groupe. Suite au procès en appel en octobre 2012, l'une des membres, Ekaterina Samoutsevitch, est remise en liberté. Les deux autres doivent attendre jusqu'à décembre 2013 pour être libérées en vertu d'une amnistie accordée par Vladimir Poutine, à la veille des Jeux Olympiques de Sotchi ${ }^{14}$.

La question qui se pose est de savoir quel est le sens social de cet événement construit dans le discours journalistique : met-il en avant le côté politique ou religieux des performances? La question est d'autant plus importante si on prend en compte le pouvoir non négligeable des médias qui, à travers l'activité de nomination et la récurrence de certains termes, peuvent finalement aboutir à leur stabilisation dans la mémoire collective d'une société donnée. Les sens ainsi actualisés peuvent, certes, varier d'un discours à l'autre ou faire l'objet de clarifications ${ }^{15}$, il n'empêche qu'une fois transmis, ils peuvent influer sur les com-

12 K.J. Rousselet, G. Favarel-Garrigues, La Russie contemporaine, Fayard, Paris 2010, pp. 355-366.

${ }^{13}$ Voir l'interview du groupe, <https://www.theguardian.com/world/2017/sep/01/pussy-riotmariya-alyokhina-russian-activist-jailed-white-house> [consulté le 28.07.2018].

${ }^{14}$ Voir K. Kurczab-Riedlich, Wowa, Wołodia, Wtadimir. Tajemnice Rosji Putina, Wydawnictwo W.A.B., Warszawa 2016, p. 626.

${ }^{15}$ Voir L. Calabrese, «Reformulation et non-reformulation du mot islamophobie. Une analyse des dynamiques de la nomination dans les commentaires des lecteurs ", Langue française 4, vol. 188, 2015, pp. 91-104. 
portements d'autres acteurs, y compris le public des médias. Comment donc le discours journalistique français, enraciné dans la réalité régie par la loi de 1905, se positionne-t-il par rapport à cet événement jugé en Russie comme contraire aux normes religieuses ? Et enfin, dans quelle mesure prend-il la responsabilité discursive de ces jugements?

\section{LA PERFORMANCE SAISIE PAR LA PRESSE : PARADIGME DÉSIGNATIONNEL DE L'ÉVÉNEMENT}

Le moment discursif initié dans la presse française ne commence que plus d'un mois après la performance, le 23 mars 2012. C'est alors que Libération parle d'une performance et d'une chanson anti-Poutine, des étiquettes qui mettent en avant le côté artistique puis politique de l'événement. Mais ce n'est qu'avec le retentissement croissant de l'affaire et surtout la prise de position successive des autorités russes que la presse française commence à s'intéresser vraiment à cette actualité russe et que de nouvelles nominations émergent en discours.

L'analyse des fréquences nous a permis de reconstituer la liste des syntagmes qui relèvent du parcours onomasiologique des énonciateurs du discours et qui forment le paradigme désignationnel de l'événement :

\begin{tabular}{|l|}
\hline prière (27 occurences) \\
\hline performance (20) \\
\hline hooliganisme (14) \\
\hline incitation à la haine religieuse (9) \\
\hline action (8) \\
\hline blasphème (8) \\
\hline sacrilège (n.) (11) \\
\hline crime (8) \\
\hline chanson (6) \\
\hline faute éthique (4) \\
\hline vandalisme (4) \\
\hline couplet (2) \\
\hline scandale (3) \\
\hline insulte (1) \\
\hline spectacle (1) \\
\hline danse/danses (1/1) \\
\hline happening (1) \\
\hline
\end{tabular}


À première vue, on observe que le paradigme est hétérogène et qu'il est constitué d'unités qui s'inscrivent dans des champs sémantiques différents : celui de l'artistique (performance, happening, chanson, spectacle, couplet, morceau, concert, danse), du légal/éthique (crime, faute éthique, hooliganisme, vandalisme) et du religieux (prière, sacrilège, blasphème, incitation à la haine religieuse). Les relations sémantiques qui se tissent entre les éléments du paradigme sont de natures différentes. On y observe les relations hyperonymiques qui lient performance à chanson, spectacle, danse ou crime à vandalisme et hooliganisme; la relation de co-hyponymie entre performance et happening ou sacrilège et blasphème, les relations méronymiques qui lient chanson à couplet, mais aussi des caractérisations (" désignations qualifiantes ») qui construisent une image équivoque de l'événement (chanson et blasphème ou prière) ${ }^{16}$. On pourrait, certes, envisager cette diversité comme témoignant de la multidimensionnalité du référent, qui remplit simultanément un certain nombre de conditions pour pouvoir entrer dans des catégories diversifiées ${ }^{17}$. Mais, comme on le verra bientôt, elle peut également être considérée en termes de conflit dénominatif, voire de « combat lexico-idéologique ${ }^{18}$, ce qui implique une mobilisation de plusieurs sources énonciatives pour adopter des attitudes idéologiques contradictoires. En effet les emplois dans lesquels un nom désignant l'événement culturel mobilise dans son contexte le lexique politique [concert + contre + désignant du courant politique] ou bien religieux [concert + en faveur de + désignant de l'adhérant d'une religion] ne manquent pas dans la presse occidentale :

(1) En Allemagne, 50000 personnes à un concert contre l'extrême droite (Le Monde, 3.09.2018)

(2) Publicité pour un concert en faveur des chrétiens d'Orient : la RATP cherche un compromis (Le Monde, 4.04.2015)

La mise en relation des sèmes qui évoquent ces deux sphères implique en revanche une relation de synonymie discursive entre les mots qui en sont porteurs et donc une collusion des valeurs politiques et religieuses dans le monde référentiel réel ${ }^{19}$, ou un conflit linguistique au niveau des signifiants/étiquettes du référent en question.

16 Voir S. Moirand, op. cit., pp. 27-28.

${ }^{17}$ Si l'on adoptait une conception référentialiste du sens, voir G. Kleiber, « Sens, référence et existence : que faire de l'extra-linguistique ? », Langages 127, 1997, p. 21. Dans la présente étude, nous nous penchons vers la conception constructiviste, où «le sens se définit en termes de propriétés des scènes verbales, construites par la parole, sans faire directement appel à la situation référentielle correspondante » (B. Victorri, « Langage et géométrie : l'expression langagière des relations spatiales », Revue de Synthèse 124, Springer Verlag/Lavoisier, 2003, p. 124).

${ }^{18}$ G. Petiot, «Voile, tchador ou foulard ? Problèmes de dénomination dans des discours médiatiques », Les Carnets du Cediscor 3, 1995, p. 61.

19 Voir la note 15. 


\subsection{PERFORMANCE : UNE CRÉATION ARTISTIQUE ET ENGAGÉE}

L'ensemble des expressions citées ci-dessus permet tout d'abord d'attribuer au référent le statut ontologique d'événement. Les noms tels que performance, happening, concert ou spectacle assurent ce repérage événementiel, car il est possible de les associer au verbe avoir lieu. En outre, leur fonctionnement possible en tant que complément de lieu permet de saisir l'événement comme « duratif et programmé $»^{20}$. Les unités impliquant une participation humaine apportent de plus simultanément l'argument sémantique de « responsabilité ». L'identification de l'agent est possible surtout grâce au complément génitif nominal en $d e^{21}$ ou déterminant possessif à valeur anaphorique :

(3) La performance des Pussy Riot (Le Monde, 22.08.2012)

(4) La performance des jeunes femmes (Libération, 27.07.2012)

(5) la performance de sa [le père d'Ekaterina Samoutsevitch] fille et de ses acolytes (Libération, 18.08.2012)

(6) leur performance (Libération, 3.08.2012)

Outre le caractère non fortuit de l'événement, les nominations en question permettent de saisir celui-ci en termes de création artistique. On y retrouve donc des termes qui mettent en scène le caractère auditif (chanson, concert, couplet, morceau) et/ou monstratif de l'événement (spectacle, happening, performance). Ayant pour trait commun le sème [+ événement artistique], elles pourraient être considérées comme résultat d'une catégorisation neutre. Or, le choix de l'expression la plus fréquente, performance (22 occurrences), et de son co-hyponyme happening est déjà loin d'être " innocent ». Ces deux notions n'ont acquis leurs acceptions actuelles qu'au $\mathrm{XX}^{\mathrm{e}}$ siècle, et semblent apporter une connotation « engagée » à l'événement. Le terme performance appartient simultanément à plusieurs champs thématiques (psychologique, technologique, sportif, politique, linguistique) et exprime une idée d'action, dénotée par sa base verbale (empruntée probablement à l'anglais : to perform « réaliser, accomplir », TLFi). Sur le plan artistique, cette notion désigne une forme de création artistique qui dépasse les frontières des modèles artistiques traditionnels. Selon Maud Hagelstein, la

${ }^{20}$ R. Huyghe, « Noms d'objets et noms d'événements : quelles frontières linguistiques ?», Scolia 26, Université des sciences humaines, Strasbourg 2012, p. 12, <https://hal-univ-diderot.archives-ouvertes.fr/hal-00975571/document> [consulté le 5.09.2018]. Ce caractère n'est pas attribuable à l'anglicisme happening qui désigne une forme de création artistique spontanée (Larousse, en ligne). Toutefois, le caractère hapaxique du mot dans le corpus (une seule occurrence) n'influe pas considérablement sur le sens social de l'événement et n'empêche pas de le percevoir comme étant planifié.

${ }^{21} C f$. M. Koptjevskaja-Tamm, «Adnominal possession in the European languages: form and function », STUF - Language Typology and Universals 55(2), 2012, pp. 141-172 (DOI : 10.1524/ stuf.2002.55.2.141[consulté le 10.09.2018]); E. Née, «(L') insécurité ou de la fabrication d'un objet consensuel dans le quotidien Le Monde ", [dans :] G. Cislaru et al., L'acte de nommer. Une dynamique entre langue et discours, Presses Sorbonne Nouvelle, Paris 2007, pp. 117-133. 
performance en tant que genre artistique se distingue par son caractère éphémère, non reproductible, et par le fait de faire « quelque chose advenir ». Dans le processus artistique, les créateurs mobilisent les objets, les gens ou les faits de la vie quotidienne, en mariant en même temps «l'art et la vie». Qui plus est, la performance exige la participation d'un spectateur dans la construction du sens de l'œuvre ${ }^{22}$. Le choix préférentiel du terme performance ${ }^{23}$ dans la presse française permet donc de saisir l'événement non seulement dans sa dimension artistique, mais de l'enraciner profondément dans la réalité sociopolitique. Intuitivement, on pourrait attribuer à performance le sème [+ art engagé], le contexte extralinguistique permettant souvent d'identifier cette forme d'action à une forme de contestation de la réalité ${ }^{24}$. L'analyse des termes classificateurs co-occurrents de performance ainsi que de ses reformulations au niveau micro permet de vérifier cette hypothèse :

(7) performance

(a) la performance des Pussy Riot (1)

la « performance» des Pussy Riot (1)

la performance de sa fille et de ses acolytes (1)

la performance des jeunes femmes (1)

leur performance (1)

leur performance dans la cathédrale du Christ-Sauveur, en février à Moscou (1)

leur performance du 21 février dernier (1)

la performance dans la cathédrale du Christ-Sauveur (1)

(b) la performance Sainte Vierge, chasse Poutine ! du 21 février à la cathédrale du ChristSauveur de Moscou (1)

la performance « Vierge Marie, chasse Poutine ! » (1)

(c) une performance artistique de trente secondes (1)

une performance plutôt marginale d'art provocateur (1)

une performance [souvent jugée] « idiote », « immature », « déplacée » (1)

une performance [souvent jugée] « idiote » et " sans grand intérêt» (1)

[décrite plusieurs fois comme] une série de "gigotements démoniaques », dans des tenues inappropriées, épaules découvertes et vêtements de couleurs criardes (1)

[perçue comme] une insulte aux sentiments religieux des croyants (1)

éléments de « sacrilège » $(1)$

[Kirill I ${ }^{\text {er }}$, patriarche de Moscou et de toutes les Russies a qualifié la performance de] « sacrilège »

[Vsevolod Tchapline, le porte-parole du patriarcat, dénonçait] « un crime pire qu'un meurtre » (1)

[que Tolokonnikova a qualifiée de ] « faute éthique » (1)

${ }^{22}$ M. Hagelstein, « Les paradoxes de l'art performance », Culture : le Magazine Culturel de l'Université de Liège, 2012, <http://culture.uliege.be/jcms/prod_922017/fr/les-paradoxes-de-l-artperformance?part=1> [consulté le 26.08.2018].

${ }^{23}$ Nous ne prenons en compte que les emplois en usage, où le mot employé renvoie à un objet du monde ( $c f$. J. Authier-Revuz, "Repères dans le champ du discours rapporté », L'Information Grammaticale 55, 1992, pp. 38-42). Ceux-ci constituent la quasi-totalité de tous les emplois du mots. Le terme n'apparait qu'une seule fois en mention : « La "performance" des Pussy Riot n'avait duré que quelques minutes [...]» (Le Figaro, 27.04.2012).

${ }^{24}$ Les exemples ne manquent pas, telles les créations de Banksy, Marina Abramović ou encore des performances féministes, toutes étant une forme de rébellion. 
Sur vingt emplois du mot, neuf sont en co-occurrence avec des groupes prépositionnels qui assurent le cadre situationnel de l'événement en apportant une information sur le lieu, le temps et l'origine de l'œuvre (7a). À deux reprises, le cotexte permet d'attribuer à l'événement un caractère explicitement politique : tout d'abord, à travers la phrase épithète $(7 \mathrm{~b})$ postposée au nom en question. Par son caractère apostrophique, par le destinataire visé (l'instance religieuse) et le souhait qui engage la fonction conative, cette interpellation partage les traits d'un genre issu du discours religieux, à savoir la prière (voir section 3.2.). On notera cependant que malgré les similitudes, l'effet visé de l'énoncé (revendication politique) et son cadre situationnel (le fait d'intervenir brusquement, en cagoule, sur l'autel d'une église ${ }^{25}$ ), marquent un détournement dialogique ${ }^{26}$ et font plutôt entendre un souhait de nature politique. Notons aussi que cette phrase injonctive est dans son premier emploi l'incipit de la chanson, attribuable à ses auteurs - locuteur et énonciateur en coïncidence ${ }^{27}$. Pourtant, son fonctionnement syntaxique (épithète) et énonciatif dans ce discours permet de l'inscrire dans la catégorie des titres et de faire entendre également la voix du journaliste. Le fait d'assimiler les premiers vers de la chanson à un titre semble relever d'une pratique journalistique dont l'objectif est d'identifier et de condenser le « contenu » de l'événement, mais aussi, dans ce cas précis, de mettre en avant son caractère conflictuel. De la sorte, le co-occurrent de performance, acquérant ici une fonction d'élément paratextuel à visée 《 séductive $»^{28}$, permet d'envisager l'événement en tant que susceptible d'attirer les regards du public. Il en va de même pour les reformulants à forte charge évaluative, introduits à travers les formes verbales relevant de l'actualisation modale. Comme on le voit dans (7c), ils s'inscrivent en majorité dans les propos tenus par les autorités russes, dont la voix semble parfois « se dissoudre » dans celle, anonyme, d'une classe plus large non explicitement déterminée (celle des profanes ?) (formes participiales jugée ou décrite dépourvues de complément d'agent, adverbe et locution adverbiale souvent/plusieurs fois guidant vers une lecture généralisante). Les formes verbales (juger, considérer, qualifier, décrire) semblent à première vue masquer la frontière entre l'opinion et l'appréciation ${ }^{29}$. Ce sont les propriétés accordées à la cible qui nous orientent vers une lecture

${ }^{25}$ Enregistrement de la performance disponible en ligne $:<$ https://www.youtube.com/watch? $\mathrm{v}=$ grEBLskpDWQ> [consulté le 2.09.2018].

26 J. Bres, « Marques de dialogisme », [dans :] Termes et concepts pour l'analyse du discours, C. Détrie et al. (dir.), Honoré Champion, Paris 2017, p. 97 ; A. Nowakowska, « De la confession catholique à la confession cathodique dans les émissions de reality-show... Du détournement d'un genre », [dans :] Actes du colloque "Les mises en scène du discours médiatique", juin 2007, Université Laval, Québec 2008, <http://asl.univ-montp3.fr/L308-09/MCC6/E62SLMCC1_MTV/cours/Nowakowska. Mise_en_page2.pdf $>$ [consulté le 5.09.2018].

${ }_{27}$ Cf. Fauré L., « Locuteur », [dans :] Termes et concepts..., pp. 207-209.

28 G. Genette, Seuils, Éd. Du seuil, Paris 1987, pp. 96-97.

${ }^{29}$ Selon Patrick Charaudeau, la différence entre les deux modalités réside dans la nature de l'opération impliquée : l'opinion relève de l'ordre intellectuel et implique donc la raison ; l'appréciation, 
appréciative et/ou de jugement, les deux ordres relevant de l'évaluation ${ }^{30}$. Les énonciateurs s'appuient sur les normes et les valeurs esthétiques et éthiques/morales pour exprimer leur attitude envers un artefact (performance), ce dernier étant le résultat d'une action humaine. Par la médiatisation des paroles qui mettent en œuvre le système des normes et des valeurs, on voit émerger le rôle sociétal ambigu de la performance.

\subsection{PRIÈRE, CRIME, VANDALISME : ENTRE LE SACRÉ ET LE PROFANE}

Une lecture religieuse de l'événement semble être suggérée par le mot le plus fréquent du paradigme, prière :

(8) prière

(a) une prière antiPoutine (5)

une « prière anti-Poutine » (1)

une « prière » contre Vladimir Poutine (1)

(b) une prière punk antiPoutine (2)

une « prière punk » anti-Poutine (1)

une « prière punk » intitulée « Marie mère de Dieu, chasse Poutine !» (2)

une « prière punk » qui disait « Vierge Marie, chasse Poutine » (1)

une prière punk intitulée « Vierge Marie, chasse Poutine » (1)

(c) une/la/leur « prière punk » (10)

trente secondes de leur prière punk (3)

Au premier abord, l'emploi de ce mot semble conférer à l'événement une dimension religieuse, cette dernière étant également présente dans la structure de l'énoncé à l'église ${ }^{31}$ (recours au vocatif, convocation de la divinité, modalité impérative) et dans le lieu où il s'est produit. Prière inscrit l'événement dans un mode de souhaitable, par recours à un rituel dans lequel l'orant invoque une divinité pour la faire agir. Comme le souligne Olivier Boulnois, ce qui est énoncé dans la prière est d'ordre divin ${ }^{32}$. Comme on le voit, dans plus de la moitié

quant à elle, met en place l'affectif (P. Charaudeau, Grammaire du sens et de l'expression, Hachette, Paris 1992, pp. 604-606).

${ }^{30}$ Nous nous appuyons ici sur le modèle cognitif de l'évaluation Appraisal, qui regroupe l'évaluation en trois sous-systèmes : Attitude/Affect (le terme diffère selon les traductions) qui regroupe les sentiments de l'énonciateur ; le Jugement, c'est-à-dire l'évaluation des comportements et des personnes selon les normes; et l'Appréciation qui est une évaluation des choses (sur les faiblesses de cette théorie, voir A. Jackiewicz, Études sur les discours évaluatifs et d'opinion, l'Harmattan, Collection Humanités Numériques, Paris 2016).

${ }^{31}$ Rapporté dans la presse française sous forme de traduction et donc relevant de la récursivité dialogique (la voix journalistique rapporte l'énoncé du collectif en rapportant d'abord — implicitement - l'énoncé du traducteur).

${ }^{32} \mathrm{O}$. Boulnois, « Quand la réponse précède la demande. La dialectique paradoxale de la prière chrétienne ", Revue de l'histoire des religions 2, 1994, pp. 167-186, <https://doi.org/10.3406/rhr. 1994.1410> [consulté le 28.08.2018]. 
de ses emplois du mot, les co-occurents situent le souhaitable dans le monde du politique. Ce n'est donc pas à une performance, ou encore moins à une manifestation civique, mais à la prière qu'est attribuée une performativité, un pouvoir de réaliser l'attente des citoyens. Cette inscription de la prière dans le contexte du pouvoir présidentiel peut se traduire par : i) l'état subjectif du priant, à savoir son impuissance face au réel, et donc, son recours au divin, ii) une stratégie consciente d'imbrication du politique et du religieux. Étant donné le détournement du genre de la prière qui se réalise déjà au niveau des conditions de production de l'événement et le fait qu'au niveau discursif, l'équivalent sémantique le plus fréquent de prière est performance et que son co-occurrent préférable dans le cotexte du droit est la préposition/le préfixe contre/anti + nom du président, le sens social de l'événement s'éloigne du domaine religieux pour se rapprocher du politique. Ajoutons aussi que dans 13 cas, le mot prière est accompagné de l'épithète punk, ce qui oriente sa lecture vers l'univers de l'art, mais aussi — par la présence des sèmes connotatifs [ + contestataire] [+ social/politique] — vers le désaccord avec l'ordre existant. L'éloignement de la dimension sacrée est également signalé au niveau énonciatif et pris en charge par le journaliste-locuteur $\mathrm{L}_{1}$. Celui-ci, par le biais d'une modalisation autonymique d'emprunt ${ }^{33}$ qui englobe 16 emplois du mot, propose un commentaire réflexif sur l'emploi du mot prière ou des $\mathrm{SN}$ prière punk/prière anti-Poutine. Dans tous les cas, les guillemets encadrants contestent une lecture strictement religieuse de l'événement. On pourrait, certes, supposer que ce procédé est une forme de dialogisme interdiscursif qui remet en question l'emploi de la tournure contradictoire dans des discours antérieurs. On n'observe cependant aucune explication de la source de la parole ni la présence de la source même, car c'est dès le début du moment discursif que la tournure entre guillemets apparaît dans le discours. L'emploi de cette forme peut donc être considéré, dans un premier temps, comme une marque d'écart entre le signe et la réalité qu'il est susceptible de refléter, de «la non-coïncidence entre les mots et les choses » ${ }^{34}$ qui se réalise pourtant sans aucune glose métaénonciative. En second lieu, les guillemets peuvent découler de la réflexion du locuteur sur l'emploi de l'oxymore qui tente de réconcilier l'ordre religieux avec un individu - ici un groupe - se confiant volontairement à la providence de Dieu, à l'ordre (a)politique de contestation de toute autorité. Or, si l'événement se rapproche de ce premier, ce n'est que par le décor dont les locutrices $1_{1}$ sont metteuses en scène, le vocatif à visée directive, adressé à une sainte de l'Église apparaissant dans l'hétérogénéité montrée du discours. La lecture religieuse de l'événement est affaiblie par le locuteur $\mathrm{L}_{1}$ — identifiable à la voix du journaliste — par l'emploi des guillemets et, au

33 Que nous comprenons ici, à l'instar de Jacqueline Authier-Revuz, comme une marque dialogique qui relève de la modalisation en discours second, où l'énonciateur emploie un élément X en usage et en mention à la fois ( $c f$. J. Authier-Revuz, op. cit., p. 39).

34 J. Authier-Revuz, « Non-coïncidences énonciatives dans la production du sens », Linx 19, 1988, pp. 25-28. 
niveau syntagmatique, par des co-occurrents qui apportent le sens second : le qualificatif punk et les épithètes formés de morphèmes de négation qui précèdent le patronyme et apportent le sème de [+ contestation, + hostilité, + lutte $]$.

L'événement prend de l'ampleur au mois de juillet, quand le tribunal de Moscou décide de prolonger la détention des trois membres du groupe. La prise de position face à l'action du groupe est fortement médiatisée dans la presse française, et de nouveaux désignants, attribués toujours à des officiels russes, sont introduits en discours. Les trois notions qui semblent alors situer l'événement dans le domaine politique, sont vandalisme (4 occurrences), hooliganisme (14 occurrences) et crime (8 occurrences) :

(9) Les trois chanteuses sont accusées d'actes de vandalisme motivé par la haine religieuse. (Le Monde, 10.08.2012)

(10) Inculpées pour hooliganisme, les jeunes femmes risquent jusqu'à sept années de prison. (Le Figaro, 31.07.2012)

(11) C'est un " crime lourd », rétorque le procureur, avant de requérir trois ans d'emprisonnement en colonie pénitentiaire. (Le Figaro, 8.08.2012)

Si ces trois termes fonctionnent en discours en tant que coréférants in $a b$ sentia, ils ne désignent que différents types d'infractions pénales à des degrés de gravité différents. On observe donc qu'une relation d'hyperonymie se tisse entre les deux termes se référant à des contraventions ${ }^{35}$ et crime, qui désigne les infractions les plus graves et qui contribue, quant à lui, à la construction du sens social de l'événement.

Il est également à noter qu'aucun de ces termes n'est employé que dans sa seule dimension politique :

(12) Les trois jeunes femmes du groupe Pussy Riot ont été condamnées pour « hooliganisme » et « incitation à la haine religieuse ». (Le Figaro, 18.08.2012)

35 Il est à signaler que le mot hooliganisme peut acquérir dans la presse française un sens différent par rapport à son équivalent russe, censé être le vrai objet de la parole médiatique. Comme le note Alice Krieg, le fait de rapporter les paroles dans une langue autre que la langue d'origine aboutit à un décalage sémantique. À cause du découpage différent de la réalité dans les langues et de la mémoire différente des usages, le déjà-dit qui pèse sur l'expression «nettoyage ethnique » n'est probablement pas identique à ceux qui pèsent, diversement, sur les plus-ou-moins-synonymes que sont ethnic cleansing en anglais, ethnische Säuberung en allemand, limpieza étnica en espagnol, pulizia etnica en italien, ou ètnitchiéskaia tchistka en russe (A. Krieg, " Analyser le discours de presse », Communication 20/1, 2000, URL : <http://journals.openedition.org/communication/6432> ; DOI : 10.4000/communication.6432 [consulté le 25.08.2018]). Bien qu'il soit lexicalisé en français, le mot hooliganisme, apparaît dans la majorité des cas (13 sur 14) en modalisation autonymique d'emprunt. La mise entre guillemets provient tout d'abord du fait de citer une autre instance énonciative, mais elle signale en même temps le contexte pénal russe. Les guillemets, faisant écho à la première acception du mot hooligan (en U.R.S.S. ou dans les pays de l'Est, jeune qui était jugé coupable de comportements asociaux et d'hostilité au régime, Larousse en ligne), semblent définir le concept selon la mémoire de son usage en langue russe, mais aussi restreindre une interprétation fréquente en français, liée aux actes de vandalisme des pseudo-supporteurs lors des compétitions sportives. 
(13) [...] un tribunal russe vient de condamner pour « vandalisme motivé par la haine religieuse » trois jeunes femmes du groupe punk Pussy Riot [...] (Le Monde, 20.08.2012)

(14) Le patriarcat avait estimé que ce « crime » était « pire qu'un meurtre » (Le Figaro, 18.08.2012)

Sur 14 emplois du mot hooliganisme, le mot est coordonné à six reprises avec le SN incitation à la haine religieuse (12). Cette catégorisation permet de considérer les deux aspects — politique et religieux — comme deux facettes équivalentes du même événement. À trois reprises, le même terme, ainsi que vandalisme sont définis à travers un GP contenant haine religieuse / haine de la religion (13). Le sens du participe passé épithète motivé attribue la primauté au facteur religieux, la haine religieuse étant présentée comme source d'un comportement interdit par la loi. C'est alors la distance syntaxique très proche des $\mathrm{SN}$ en question qui influe sur la référence de vandalisme et hooliganisme et qui permet de saisir l'événement sous ses deux aspects. En ce qui concerne crime, le sens lexical même du mot permet de cacher les enjeux politiques et religieux à la fois ${ }^{36}$, même si le cadre énonciatif semble privilégier une lecture religieuse, en situant l'action du groupe comme soumise à l'évaluation d'une autorité orthodoxe. À cela s'ajoutent les représentations lexicales des actes et des paroles sacrilèges :

(15) Kirill I ${ }^{\text {er }}$, patriarche de Moscou et de toutes les Russies, a qualifié la performance de « sacrilège », tandis que Vsevolod Tchapline, le porte-parole du patriarcat, dénonçait « un crime pire qu'un meurtre ». (Le Figaro, 17.08.2012)

(16) Quand la justice russe traque le « sacrilège » (titre, Le Figaro, 14.07.2012)

(17) Pour cet acte considéré comme doublement sacrilège, trois d'entre elles, Nadejda Tolokonnikova, 22 ans, Maria Alekhina, 24 ans, et Ekaterina Samoutsevitch, 29 ans, ont été arrêtées et placées en détention. (Le Monde, 9.08.2012)

(18) Dans son réquisitoire, mardi, le procureur avait déclaré que le blasphème est un crime aussi grave que la diffamation. (Libération, 9.08.2012)

(19) Blasphème et rébellion en Russie (titre, Le Figaro, 17.04.2012)

(20) [...] une avocate de la défense, Violetta Volkova. Et de s'adresser au procureur : « Dans quel code pénal les accusateurs sont-ils allés chercher ces termes - sacrilège, blasphème, jambes levées avec obscénité ? ». (Libération, 9.08.212)

(21) « Certains termes utilisés, comme le sacrilège, font référence à la langue religieuse du Moyen Âge. Dans un État laïc comme la Russie, de telles formulations n'ont rien à faire dans un acte d'accusation ", s'étonne ce scientifique [le directeur du Centre d'études de la religion, Nikolaï Chabourov]. (Le Figaro, 14.07.2012)

Comme on le voit, le discours journalistique permet de retracer l'itinéraire discursif de deux mots, sacrilège et blasphème, employés d'abord par les autorités orthodoxes (15), repris par les fonctionnaires judiciaires chargés de l'instruction $(16,17,18)$, et qui deviennent enfin sujets des discours des avocats et intellectuels

${ }^{36}$ Comme l'indique le TLFi, crime désigne une « infraction grave punissable par la loi d'une peine afflictive ou infamante » et « infraction grave à la morale ou à la loi religieuse et réprouvée par la conscience ». 
favorables à la cause des musiciennes $(20,21)$. Quant à la voix des journalistes, elle opte pour une stratégie d'évaluation de l'adéquation de ces mots au référent visé : l'emploi assez systématique des guillemets pour rapporter les sources énonciatives qui sont à l'origine des termes $(15,16)$ et la thématisation de ces mots dans les titres de presse $(16,18)$ mettent en exergue la non-coïncidence entre ces mots et les choses. Le positionnement des journalistes se manifeste également dans la structure de (16), où la prédication est effectuée sur un objet relevant de l'ordre religieux par un agent lié au monde politique. La conjonction quand à valeur causale ainsi que les guillemets assurent une forte valeur prédicative aux deux arguments et mettent en avant une collusion de deux ordres qui deviennent inséparables. Les tendances désécularisantes sont aussi signalées par des tiers (20, 21) et s'expriment en plus dans la récursivité dialogique, puisque les deux termes, originairement employés par les officiels religieux se voient actualisés dans le discours judiciaire.

Le débat autour le caractère de l'événement voit son couronnement dans la presse aux mois de juillet et août 2012. C'est alors qu'émerge en discours une étiquette à valeur événementielle, affaire (des) Pussy Riot (22 occurrences). Cette anaphore résomptive évoque les discours anérieurs liés aux protagonistes, tout en masquant d'autres coordonnées (caractère politique/religieux de l'événement). Comme l'observent Olivier Turbide et al., l'emploi de ce mot impose une lecture dialectique : «l'affaire surgit lorsqu'il y a expressions multiples et contradictoires sur un fait $»^{37}$. L'obscurité sémantique du mot, mais aussi une très faible présence du terme événement ( 2 occurrences) font que le vrai objet du discours devient non pas le fait même, mais le débat qui a éclaté autour de cet événement.

\section{CONCLUSION}

L'examen du traitement médiatique de l'événement en question a permis de dégager plusieurs procédés qui ont abouti à la construction du sens social de celui-ci. Tout d'abord, l'hétérogénéité du paradigme désignationnel, la diversité énonciative et une forte présence des formes axiologiques reflètent le caractère ambigu de cet événement qui transgresse les frontières de l'art, du politique et du religieux. L'équivalence discursive entre des désignants qui sont reliés par une relation d'antonymie [performance anti/contre + patronyme présidentiel/blasphème $]$ contribue par la suite à créer l'image d'un État régi par les normes religieuses.

Quant aux stratégies discursives de la presse, on observe que les quotidiens français visent à transmettre l'expérience de l'Autre en reproduisant les dires qui

37 O. Turbide, D. Vincent, E. Kavanagh, « Repères méthodologiques pour l'analyse des discours sociaux. Lorsqu'un tweet devient une affaire », [dans :] D. Londei et al., Dire l'événement. Langage, mémoire, société, Presses Sorbonne Nouvelle, Paris 2013, p. 25. 
assimilent la performance du groupe à un outrage au sacré. En même temps, la voix des journalistes se distancie de la lecture religieuse de l'événement, en optant systématiquement pour les guillemets, les épithètes qui accompagnent les SN « religieux » et qui, sans être marqueurs de glose, assurent une lecture politique du fait, ou enfin les commentaires métalinguistiques (X est considéré/jugé comme) qui expriment la distance par rapport aux dires des officiels russes. Qui plus est, les modalisations autonymiques d'emprunt signalent un " désaccord tacite » des journalistes envers un régime politico-religieux in statu nascendi. Le positionnement est plus visiblement exprimé dans les titres qui mettent en avant la collusion du pouvoir et du religieux. La performance elle-même n'est qu'un prétexte : par son caractère polémique, elle se transforme en objet d'évaluation et aboutit à une dichotomisation des positions pour enfin s'enraciner dans la mémoire discursive non pas comme un événement, mais comme une affaire. Une affaire qui finira d'ailleurs, un an plus tard, par l'adoption par la Douma de la loi sur le blasphème ${ }^{38}$.

\section{BETWEEN THE SACRED AND THE PROFANE: THE FRENCH JOURNALISTIC DISCOURSE ON THE PUSSY RIOT AFFAIR}

Summary

On 21 February 2012, three women of the well-known punk rock band Pussy Riot organized a performance in the Cathedral of Christ the Savior in Moscow. In consequence of the exhibition at this religious place, the women were convicted for "vandalism" and "incitement to religious hatred."

The aim of this article is to examine the social meaning of this event constructed and transmitted in the French journalistic discourse. It highlights the role of religion that derives from the event and contributes to the image of contemporary Russia. In the second step, the article deals with the discursive practices and positions of the journalistic voice facing this event.

Key words: journalistic discourse, nomination, Pussy Riot, Russia.

${ }^{38}$ Cf. $<$ http://www.lefigaro.fr/international/2013/06/12/01003-20130612ARTFIG00308-la-douma-russe-s-attaque-au-blaspheme-et-a-lapropagande-homosexuelle.php> [consulté le 10.09.2018]. 